Document downloaded from:

http://hdl.handle.net/10251/158836

This paper must be cited as:

Paz-Yépez, C.; Peinado, I.; Heredia Gutiérrez, AB.; Andrés Grau, AM. (2019). Lipids digestibility and polyphenols release under in vitro digestion of dark, milk and white chocolate. Journal of Functional Foods. 52:196-203. https://doi.org/10.1016/j.jff.2018.10.028

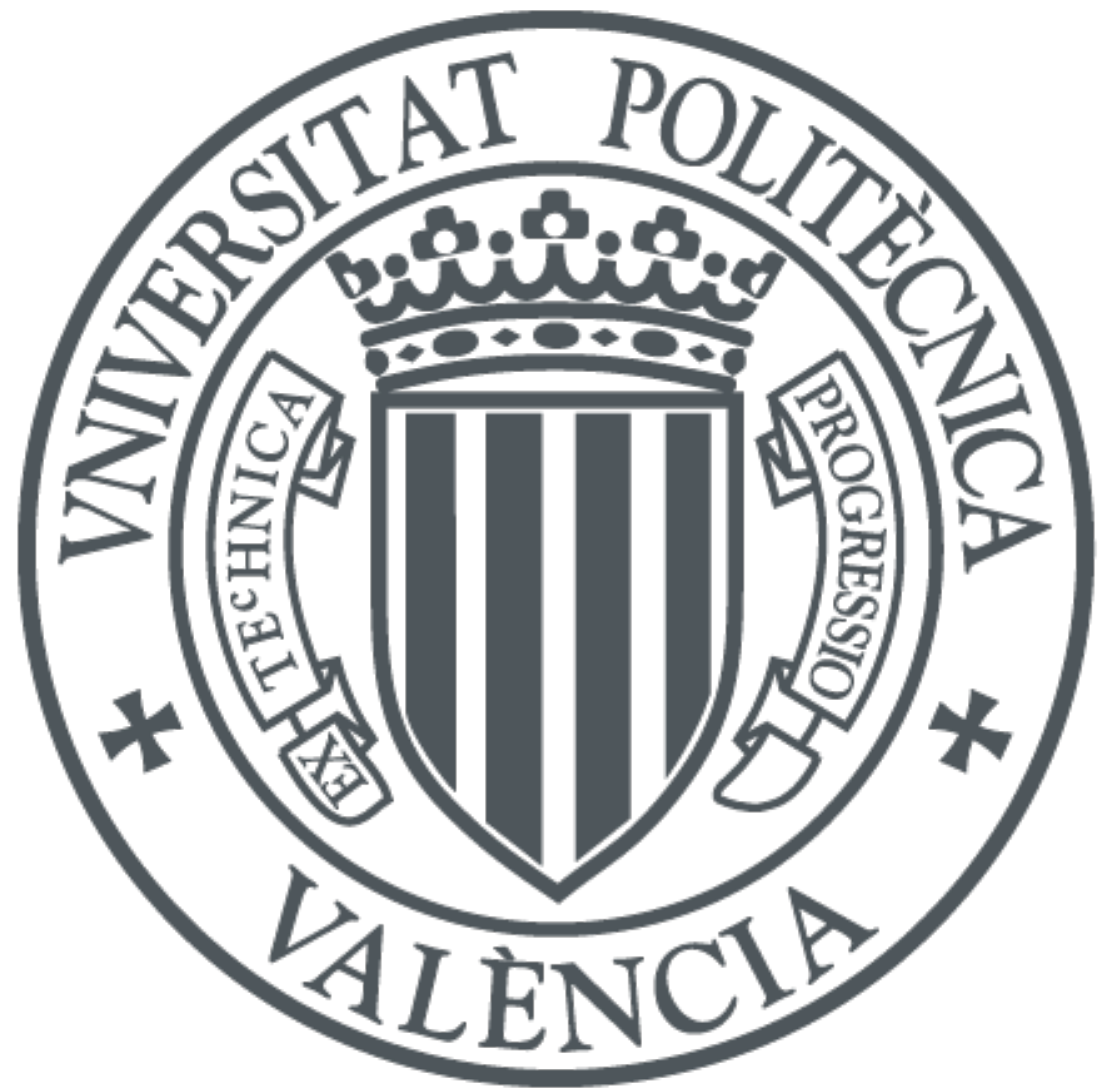

The final publication is available at

https://doi.org/10.1016/j.jff.2018.10.028

Copyright Elsevier

Additional Information 


\title{
Lipids digestibility and polyphenols release under in vitro digestion of
} dark, milk and white chocolate.

\author{
Carolina Paz-Yépez ${ }^{* 1}$, Irene Peinado ${ }^{1}$, Ana Heredia ${ }^{1}$ and Ana Andrés ${ }^{1}$
}

1 Universitat Politècnica de València, Research Institute of Food Engineering for Development, P.O. Box: 46022, Valencia, Spain *e-mail corresponding author: cpazyepez@gmail.com

\section{ABSTRACT}

This study evaluated the influence of intestinal conditions on lipolysis and polyphenols release and bioaccessibility in dark, milk and white chocolates. Chocolates were in vitro digested under different intestinal conditions of $\mathrm{pH}(6$ and 7), bile concentration (1 and $10 \mathrm{mM})$ and pancreatic concentrations $(1,000-3,000 \mathrm{LU}$ / g Fat). The lipolysis varied from 300 to $500 \mathrm{mg}$ FFA / g fat in dark chocolate and ranged between 600-1000 mg FFA / g fat in both, milk and white. Polyphenols release in dark chocolate (up to $12 \mathrm{mg} \mathrm{GA} / \mathrm{g}$ ), seems to be related to the absence of dairy compounds. Finally, no effect of intestinal $\mathrm{pH}$ or biliary concentration was found on the lipolysis in digested dark and milk chocolates. The oral pancreatic supplementation, however, was crucial to lipolysis and polyphenols release in all chocolates, even if no differences were found on these parameters from $2,000 \mathrm{LU} / \mathrm{g}$ fat.

Keywords: in vitro digestion; pancreatic insufficiency; chocolate; lipolysis; polyphenols. 


\section{Introduction}

Chocolate is a fat continuous matrix within which there are particles of cocoa powder, sugar, and in the case of milk chocolate, milk powder. Besides the high fat content of chocolate, generally exceeding $30 \mathrm{~g}$ of fat per $100 \mathrm{~g}$ of food (United States Department of Agriculture, 2018), it has lately gained attention mainly due to its main component, cocoa. In fact, cocoa is well known for its lipid and antioxidant properties. Cocoa butter, has a lipid profile characterized mainly by oleic, stearic $(\approx 34 \% \mathrm{w} / \mathrm{w})$ and palmitic $(\approx 27 \% \mathrm{w} / \mathrm{w})$ acids, followed by minor fractions of linoleic, arachidic, palmitoleic, margaric, a-linoleic and myristic acids. In addition, cocoa is a polyphenols-rich product, being one of the largest known food sources of flavan-3-oles, mainly epicatechin, catechin and notably procyanidins (Beckett, 2008; Jalil \& Ismail, 2008).

In the last years, many studies have reported a relation between the consumption of cocoa derivatives, especially dark chocolate, with beneficial health effects on cardiovascular and degenerative diseases, related to the antioxidant activity of procyanidins (Almoosawi, Tsang, Ostertag, Fyfe, \& Al-Dujaili, 2012; Cooper, Donovan, Waterhouse, \& Williamson, 2008; Jalil \& Ismail, 2008; Keen, Holt, Oteiza, Fraga, \& Schmitz, 2005; Ortega, Reguant, Romero, Macià, \& Motilva, 2009). Most of these studies extensively reported not only the in vitro health benefits of cocoa consumption but also its effects in vivo (Jalil \& Ismail, 2008; Kurosawa et al., 2005; Matsui et al., 2005; Ramiro et al., 2005; Vinson et al., 2006). However, while most polyphenols showed antioxidant action in vitro, they did not necessarily exert antioxidant potential in vivo (Cooper et al., 2008; Jalil \& Ismail, 2008). Related to the health protective effects of cocoa polyphenols, it is important to point out the modifications that undergo the processing of cocoa such as seed fermentation, roasting, nib-grinding, alkalizing, tempering, molding or enrobing, cooling, and packing (Beckett, 2008; Ortega et al., 2009; Wollgast \& Anklam, 2000) might modify the chemical features of polyphenols compounds and therefore, their effects on health. Besides, it is also important to consider the interaction that polyphenols might exert with other components within the food matrix, such as macromolecules, since these can have significant effects on their bioaccessibility and bioavailability (Jakobek, 2015). For instance, the interaction between lipids and polyphenols has been reported in literature. In a previous study of in vitro digestibility of cocoa polyphenols in a matrix containing fat, Ortega et al. (2009) suggested that the fat content in the cocoa liquor could have a protective effect on cocoa polyphenols because it enhances the micellarization and stability of polyphenols during digestion. Additionally, the access of free radicals to lipid molecules can be hindered by the presence of polyphenols in lipid molecules, reducing the harmful caused by lipid oxidation products (Jakobek, 2015). On the other hand, it has also been found that polyphenols affected the emulsification taking place during the gastrointestinal process by increasing the droplet size and decreasing specific surface area, causing in addition 
a decrease in the activity of the gastric and/or pancreatic lipases (Shishikura, Khokhar, \& Murray, 2006; Sugiyama et al., 2007; Uchiyama, Taniguchi, Saka, Yoshida, \& Yajima, 2011).

Food components, such as polyphenols or lipids, will need to be released from their food matrix in order to be bioavailable, so they can become then absorbable (bioaccessible), meaning they can be absorbed by intestinal cells and be metabolized (Asensio-Grau, Peinado, Heredia, \& Andrés, 2018; Faulks \& Southon, 2005; Nimalaratne, Savard, Gauthier, Schieber, \& Wu, 2015). Nutrients bioaccessibility will depend on different factors related to the food itself such as food composition, its matrix, type of nutrients, and/or processing conditions (Granado-Lorencio et al., 2007; Nimalaratne et al., 2015; Pineda-Vadillo et al., 2017; Ryan, O'Connell, O'Sullivan, Aherne, \& O'Brien, 2008). In addition, individual factors such as gastrointestinal conditions $(\mathrm{pH}$, secretion and composition of the digestive fluids, transit time...) might affect the release of food bioactive compounds (Ryan et al., 2008; Whitcomb et al., 2010). All this leads to the importance of analysing to which extent not only food matrix, but other factors, can modify the stability and the bioaccessibility of bioactive compounds, in order to better understand the biological activity of food constituents (RodríguezRoque et al., 2015).

In fact, individual gastrointestinal conditions might be different among different individuals depending on their age, gender, diet, etc. (Shani-Levi et al., 2017). These differences will become even more relevant under specific digestive disorders. For instance, special attention has been addressed to rich-fat foods for dietary recommendations aimed at subjects with specific requirements of fat intake. This is the case of individuals suffering of exocrine pancreatic insufficiency (EPI). EPI is a physiological disorder characterized by a decrease of secretions of $\mathrm{Cl}^{-}$, water and $\mathrm{HCO}^{-3}$, with the consequent decrease of pancreatic and biliary secretions, causing dilation and obstruction of the pancreatic and bile ducts (Li \& Somerset, 2014). Due to alterations at pancreatic level, a deficiency in the production of digestive enzymes at the duodenum might occur, leading this, to malnutrition and some associated problems. Currently, the treatment of EPI is carried out by enzymatic substitution therapy (EST), which consists on oral administration of porcine pancreatin formulated as gastroresistant microcapsules. Actual guidelines of the supplement advise an enzyme amount of 2,000 Lipase Units LU/g fat intake, based on the overall fat content of the meals or on patients body weight (Turck et al., 2016). However, these recommendations seem, nowadays, insufficient for the efficiency of the treatment since hostfactors such as intestinal $\mathrm{pH}$, bile concentration as well as food intrinsic ones (composition, structure and lipid organization in the food-matrix among others) might have a significant impact on the efficiency of the supplement, and therefore on macronutrients digestion (Rovner, Schall, Mondick, Zhuang, \& Mascarenhas, 2013). 
In this way in vitro digestion models are considered useful tools that can be used to study the digestive properties of different food forms, helping to understand the mechanisms behind the differences observed in vivo. To the authors knowledge, there are already some studies focusing on lipids absorption and polyphenols bioaccessibility of cocoa based products (Jalil \& Ismail, 2008; Kurosawa et al., 2005; Matsui et al., 2005; Ortega et al., 2009; Ramiro et al., 2005; Vinson et al., 2006), however, in all of them, gastrointestinal conditions were simulated according to a standard healthy adult. Therefore, the aim of the present study was to evaluate the differences between dark, milk and white chocolate in terms of lipids digestibility and bioaccessibility of total polyphenols under different intestinal conditions $(\mathrm{pH}$, concentration of bile and pancreatic enzymes). Besides, the enzymatic supplementation leading to the highest in vitro lipolysis extent under unfavourable intestinal conditions will be obtained for each chocolate.

\section{Materials and Methods}

\subsection{Raw Material}

Three types of chocolates, available in packets of $100 \mathrm{~g}$ each, were purchased from a local supermarket (Dark chocolate (D), Milk chocolate (M), White chocolate (W)), all of them form the same brand. Table 1 details the nutritional and compositional information available in the label.

\subsection{Chemicals}

a-amylase from human saliva (1000-3000 U/ mg protein) and pepsin from porcine gastric mucosa $(\geq 2,500 \mathrm{U} /$ g protein), were obtained from Sigma-Aldrich. Pancreatin from swine pancreas (Kreon® 10,000 lipase units (LU), Abbot), was kindly donated by "Hospital Universitari Politècnic La Fe" (Valencia, Spain). Each capsule contains $150 \mathrm{mg}$ of porcine pancreatic enzyme equivalent to 10,000 lipase U., 8,000 amylase $\mathrm{U}$. and 600 protease U.

The following chemicals were needed for preparation of the simulated digestive fluids: bovine bile extract, $\mathrm{KCl}$, $\mathrm{KH}_{2} \mathrm{PO}_{4}, \mathrm{NaHCO}_{3}, \mathrm{NaCl}, \mathrm{MgCl} 2(\mathrm{H} 2 \mathrm{O})_{6},\left(\mathrm{NH}_{4}\right)_{2} \mathrm{CO}_{3}$ and $\mathrm{CaCl}_{2}$ all of them from Sigma-Aldrich Chemical Company (St Louis, MO, USA). $\mathrm{NaOH}(1 \mathrm{~N})$ and $\mathrm{HCl}(1 \mathrm{~N})$, were acquired from AppliChem Panreac. For the analytical determinations, all solvents were analytical grade; Triton-X 100\%, ethanol $96 \%$ (Labkem), enzymatic kits for the analysis of free fatty acids (Roche Diagnostics, Indianapolis, IN, USA). The analytical standards oleic acid and for the content of total polyphenols, methanol, Folin-Ciocalteu reagent, $\mathrm{Na}_{2} \mathrm{CO}_{3}$, gallic acid (GA) were all from Sigma-Aldrich). 


\subsection{In vitro simulation of gastrointestinal digestion}

\subsubsection{In vitro Digestion process}

The digestion procedure used was based on the standardized static in vitro digestion method for food published by Minekus et al. (2014) with some modifications in order to simulate EPI conditions as detailed in previous studies (Asensio-Grau et al., 2018; Peinado, Larrea, Heredia, \& Andrés, 2018). The digestion fluids were prepared fresh from stock solutions, salivary (SSS), gastric (SGS) and intestinal (SIS) according to Minekus et al. (2014). Table 2 summarizes composition and amounts of the fluids used in each of the stages of the digestion process. The enzymatic activity was tested before each experiment following the protocol proposed by Carrière et al., (2000). Each experimental condition was performed in triplicate. The in vitro digestion was performed as follows:

Oral stage: chocolates samples were mechanically broken down with a mortar, and the amount of sample to be digested was weighted in order to have $0.35 \mathrm{~g}$ fat in each tube (50 mL falcon tubes). Simulated salivary fluid $(5 \mathrm{~mL})(\mathrm{SSF} ; \mathrm{pH} 8)$ at $37^{\circ} \mathrm{C}$, was added to the falcon tubes containing chocolate samples in a ratio $1: 1$ $(\mathrm{v} / \mathrm{w})$, and then they were properly homogenized and incubated for 3 minutes at $37^{\circ} \mathrm{C}$ in an incubator chamber Selecta (JP Selecta SA, Barcelona). Human a-amylase was added as a part of SSF to reach a concentration in the saliva mixture of $75 \mathrm{U} / \mathrm{mL}$.

Gastric stage: after the oral stage, simulated gastric fluid (SGF; pH 3) was added to each tube containing the oral bolus $(1: 1 \mathrm{v} / \mathrm{w})$. Pepsin was added into the SGF to reach a concentration in the gastric mixture of 2,000 $\mathrm{U} / \mathrm{mL}$. The $\mathrm{pH}$ of the mixtures was adjusted with $\mathrm{HCl}(1 \mathrm{~N})$ to $\mathrm{pH} 3 \pm 0.1$ and samples were flipped from top to bottom at 55 rpm for 120 minutes at $37^{\circ} \mathrm{C}$ using an Intell-Mixer RM-2 (Elmi Ltd, Riga, LV-1006, Latvia) and incubated in a chamber Selecta (JP Selecta SA, Barcelona). These conditions provide mechanical energy to cause the disintegration of the food matrix during gastric digestion, in a similar way as it happens physiologically.

Intestinal stage: following the gastric stage, simulated intestinal fluid (SIF; pH 7) containing bile salts (1 or 10 $\mathrm{mM})$ and pancreatin $(0 ; 1,000 ; 2,000 ; 3,000 \mathrm{LU} / \mathrm{g}$ of fat) was added in a proportion $1: 1(\mathrm{v} / \mathrm{w})$ to each tube containing the gastric chime. The $\mathrm{pH}$ of the mixtures was adjusted with $\mathrm{NaOH}(1 \mathrm{~N})$ to $\mathrm{pH} 6.0 \pm 0.1$ or $7.0 \pm$ 0.1 , depending on the experimental design. Samples were then flipped from top to bottom at $55 \mathrm{rpm}$ for another 120 minutes at $37^{\circ} \mathrm{C}$. $\mathrm{pH}$ was monitored during the digestion process and readjusted if necessary as $\mathrm{pH}$ below 5.7 might inactivate lipase activity (González-Bacerio, Rodríguez Hernández, \& del Monte Martínez, 2010; Prazeres, Garcia, \& Cabral, 1994). 
The experimental design for each type of chocolate consisted of two sets of experiments. In the first set, the amount of pancreatic enzymes was fixed at 2,000 LU/g of lipid, and the study variables consisted on different combinations of intestinal $\mathrm{pH} / \mathrm{bile}$ concentration $(\mathrm{pH} 6 / 1 \mathrm{mM}, \mathrm{pH} / 10 \mathrm{mM}, \mathrm{pH} 7 / 1 \mathrm{mM}$ and $\mathrm{pH} 7 / 10 \mathrm{mM}$ ) in order to analyse the impact of different intestinal conditions on the lipolysis and bioaccessibility of total polyphenols. In this scenario, $\mathrm{pH} 7$ and $10 \mathrm{mM}$ of bile salts would correspond to the standard intestinal conditions of a healthy adult (Minekus et al., 2014); while pH 6 and $1 \mathrm{mM}$ would correspond to the most disadvantageous scenario in

151 EPI individuals (Gelfond, Ma, Semler, \& Borowitz, 2013). In the second experimental set, intestinal conditions were fixed at $\mathrm{pH} 6$ and bile salts concentration $1 \mathrm{mM}$, and different pancreatic enzymes amounts $(0 ; 1,000$; 2,000 and $3,000 \mathrm{LU} / \mathrm{g}$ of fat) were tested in order to assess the influence of enzyme concentration. All the experiments were designed to analyse the impact of different intestinal scenarios on matrix degradation index, lipolysis extent, and total polyphenols release.

\subsection{Analytical determinations}

157 Immediately after digestion, samples were placed in ice for 10 minutes in order to stop the enzymatic reactions before performing the analytical determinations. After that, tubes were centrifuged (4,000 $\mathrm{x}$ g-force during 20 minutes, $10 \stackrel{\circ}{ } \mathrm{C})$ to separate the solid fraction from the liquid phase. After centrifugation, the total content of each tube was filtered through a metallic sieve $(1.6 \mathrm{~mm} \times 1.6 \mathrm{~mm}$ mesh) to separate out solid food particles.

161 These solid particles were rinsed twice with $5 \mathrm{~mL}$ of appropriate juice to remove any digested material. Blotting paper was placed around the metallic sieve for $10 \mathrm{~min}$ to drain residual digestion juice. The undigested chocolate particles (solid particles) were then transferred to an aluminum dish (previously weighed). The drained liquid, called from now on "liquid phase", was collected and $0.1 \mathrm{~mL}$ was used for free fatty acids analyses; the remaining liquid phase was freeze-dried $\left(-40^{\circ} \mathrm{C}\right.$ and $1.25 \mathrm{mbar}$, Telstar, Terrassa, Spain) and stored for polyphenols determination.

\subsubsection{Matrix Degradation Index (MDI \%)}

Matrix degradation Index was determined in all samples after in the vitro digestion process. This parameter represents the proportion of solids dispersed in the digestion fluids after duodenal digestion. The aluminum dish with the undigested particles was placed in a forced air oven at $60^{\circ} \mathrm{C}$ for $48 \mathrm{~h}$ until constant weight. The matrix degradation index (MDI) corresponding to the proportion of chocolate particles passing the metallic sieve and was calculated according to Lamothe, Corbeil, Turgeon, \& Britten (2012).

\subsubsection{Lipolysis extent (mg FFA/g fat)}


enzyme. Fatty acids release during digestion was estimated using a spectrophotometric assay kit (Roche Diagnostics, Indianapolis, IN, USA) in a spectrophotometer (UV/vis, Beckman Coulter) at $564 \mathrm{~nm}$ (Lamothe et al., 2012; Asensio- Grau et al., 2018; Peinado et al., 2018). Oleic acid standard was used for quantitative determination of FFA (Free Fatty acids), since it is the major fatty acid in chocolate fat (Beckett \& Stephen, 2008). Digested fat was estimated assuming the maximum release of $2 \mathrm{~mol}$ of fatty acids per $1 \mathrm{~mol}$ of triglycerides (Hunter, 2001), and results were expressed as mg of FFA, considering the average of molecular weight of oleic acid $282.47 \mathrm{~g} \mathrm{~mol}^{-1}$.

\subsubsection{Total Polyphenols content}

Total polyphenols in the digested fluids were determined by the modified protocol of the Folin-Ciocalteu assay (Singleton, Orthofer, \& Lamuela-Raventós, 1998). Freeze dried powder (digested chocolate liquid phase (50 $\mathrm{mg}$ ), and solid undigested phase (50 mg)) was extracted using methanol ( $1 \mathrm{~mL}, 30: 70$ methanol-water solution (v: v)) by means of agitation at 55 rpm, for 120 min at $25^{\circ} \mathrm{C}$ using an Intell-Mixer RM-2 (Elmi Ltd, Riga, LV1006, Latvia). The mixture was then centrifuged at 14.1 x g-force for 20 minutes (Eppendorf® Minispin $\circledast$ ). After centrifugation, methanolic extracts $(125 \mu \mathrm{L})$ were added into a $4 \mathrm{~mL}$ plastic cuvette with distilled water $(0.5$ $\mathrm{mL}$ ) and Folin-Ciocalteu reagent $(125 \mu \mathrm{L})$. After $5 \mathrm{~min}, 1.25 \mathrm{~mL}$ of $\mathrm{Na}_{2} \mathrm{CO}_{3}$ solution $(7 \%[\mathrm{w} / \mathrm{v}])$ and distilled water $(1 \mathrm{~mL})$ were added and measurements recorded after $30 \mathrm{~min}$, at a wavelength of $660 \mathrm{~nm}$. Gallic acid (0-700 $\mu \mathrm{mol}$ of gallic acid / L) was used as standard. Results were expressed as $\mathrm{mg}$ of gallic acid (GA) equivalent per gram of food.

\subsection{Statistical analyses}

Analysis of variance (simple and multivariate ANOVA) followed by Fisher LSD post-hoc tests were performed to find out the statistical significance of the type of chocolate and the intestinal variables $(\mathrm{pH}$, bile salts concentration (mM) and pancreatin amount (UL/g fat)) on MDI (\%), lipolysis extent (\%), and polyphenols release (mg GA/g) by means of Statgraphics Centurion. Differences were considered statistically significant when $p<0.05$.

\section{Results and discussion}

\subsection{Effect of intestinal conditions on matrix degradation Index (\%) of the digested chocolates}

The matrix degradation index (MDI) corresponds to the percentage of digested particles and it provides global information about the extent of different processes (solubilisation, mechanical disruption, chemical and enzymatic reactions) undergone by the food matrix during oral, gastric and intestinal stages. During digestion, food absorbs a significant amount of water, and together with the action of digestive enzymes it promotes the 
softening of the matrix and the reduction of cohesive forces; depending on the food matrix composition and structure it will result in different degradation extent (Kong \& Singh, 2009). Additionally, in fat continuous products as chocolate, softening and matrix disruption is mainly due to melting of fat as oral temperature. Table 3 shows the results of MDI of the different chocolate types after the in vitro digestion process at different intestinal $\mathrm{pH}$ and bile concentrations, as well as for 2,000 LU / $\mathrm{g}$ fat and different pancreatin amounts. MDI ranged from 50 to $75 \%$ (average value) in dark chocolate, while it reached 87 to $94 \%$ (average value) in the other two types of chocolates (milk and white chocolate). The statistical analyses revealed that neither the intestinal $\mathrm{pH}$, the bile concentration nor the amount of pancreatin had a significant effect on MDI. The significant differences in this parameter were found to depend on the type of chocolate $(p<0.001)$, being the dark chocolate the one showing the lowest values of MDI.

It is important to consider how the different ingredients in chocolates (fat, lecithin and crystalline lactose) might affect their structure and therefore, their matrix degradation when they undergo digestion. Chocolate microstructure is the result of many factors including particle size, amount and distribution of fat, type and amount of emulsifier and solid particles (Afoakwa, Paterson, Fowler, \& Vieira, 2009). Among the main components of chocolate, the most soluble is sugar, which will explain the major degradation of chocolates containing high content of this ingredient. In addition, the amount of fat will affect particle interactions, related to their distances and distribution within the food matrix (Glicerina, Balestra, Dalla Rosa, \& Romani, 2016). In their study Glicerina et al., (2016), compared the microstructural and rheological properties of dark, milk and white chocolate, revealing that dark chocolate showed a more aggregated structure, with the lowest minimum distance between particles and less open spaces (filled with fats) than milk chocolate samples. These microstructural properties would explain the lower MDI obtained in digested dark samples (Table 3) compared to white and milk samples. The MDI values of white chocolate were the highest, although very similar to the values obtained for milk chocolate samples, probably due to the similar amount of fat in both samples (Table 1). However, the more aggregated microstructure due to the presence of cocoa particles between the sugar ones in milk chocolate would explain the lower values of MDI compared to the white chocolate.

\subsection{Effect of intestinal conditions on total polyphenols release from the digested chocolates and on}

\section{their final bioaccessibility}

Figure 1 shows the polyphenols content (mg equivalent of gallic acid / $\mathrm{g}$ of chocolate) of dark, milk and white chocolate for both, solid undigested chocolate as well as digestion fluids, after oral, gastric and intestinal digestion. The effect of intestinal $\mathrm{pH}(6$ or 7$)$ and bile concentration (1 and $10 \mathrm{mM})$ on these antioxidants is also gathered in this figure. As it can be observed, the initial quantity of polyphenols seemed to be directly related to the cocoa content in the product. Of note, dark chocolate presented the highest polyphenols content, 
five-fold superior to the content found in milk chocolate and seven-fold than in white chocolate. As it can be observed, the oral stage characterised by simulated mastication and primary hydrolysis of glucids by $\alpha$ amilase, resulted in a low release of polyphenols mainly due to the short duration of this stage. Therefore, most polyphenols remain in the solid phase, i.e. in the food matrix, after simulated mastication. The acidic $\mathrm{pH}$, together with the enzymatic proteolysis carried out by pepsin on chocolate-protein $(6,7$ and $10.4 \%$ in white, milk and dark chocolate, respectively) and the mechanical disruption acting during $120 \mathrm{~min}$, were the main responsible of the increase of total polyphenols content during this gastric digestion.

It is important to point out that the polyphenols method quantifies only soluble polyphenols. According to these results, it seems that polyphenols take part in complex molecules before digestion, being released from these structures under both, gastric and intestinal conditions. In food, polyphenols are found mainly as esters, glycosides and polymers usually not absorbable in those forms, and therefore they need to be hydrolysed by digestive enzymes or intestinal microflora. Due to the low pH in the stomach, the flavonoid oligomers are transformed into smaller units. The flavon-3-ols pass intact to the duodenum in the form of aglycones. In the small intestine, deglycosylation, glucuronidation, methylation, sulphonation and hydroxylation of flavonoids occurs. Under alkaline $\mathrm{pH}$ conditions in the small intestine, the absorption of free phenolic acids occurs. Undigested polyphenols then pass into the large intestine, where colonic microflora degraded them into phenolic acids (Tarko, Duda-Chodak, \& Zajac, 2013). Different factors such as mechanical destruction, nature of the food matrix, residence time under different gastrointestinal conditions and the enzymatic action contribute positively to the physic-chemical release of the phenolic compounds, resulting in an augmentation of the total free polyphenols content. Moreover, most polyphenols are present in the liquid phase compared to those present in the solid phase after gastric and intestinal stages, and therefore they would be more bioaccessible, and hence available to be absorbed. Of note, the solubilisation and release of polyphenols in digested white chocolate reached its maximum value during the intestinal stage, being comparable to milk chocolate, despite its low initial content of polyphenols in the undigested product. Even if polyphenols content was higher at $\mathrm{pH} 7$ and $1 \mathrm{mM}$ than under the other studied conditions, a slight increase in polyphenols released within the liquid phase was observed for the highest bile concentration $(10 \mathrm{mM})$ at $\mathrm{pH} 6$. A clear effect was not found, however, depending on bile concentration for intestinal $\mathrm{pH}$ 7. This could be explained, due to the fact that the optimum pH for pancreatic enzymes ranges from 7 to 8 (González-Bacerio et al., 2010; Prazeres et al., 1994). In fact, in a previous study on milk lipolysis, Peinado et al., (2018) reported that the relevance of bile salts concentration was higher at low intestinal pHs than at higher (7 and 8 ). In this scenario, bile salts motivate lipolysis-products transportation (mainly free fatty acid and monoglycerides) from the lipid surface to the intestinal fluid and their further micellation, facilitating thus, solubilisation of digestion products such 
antioxidants (Maldonado-Valderrama, Wilde, Maclerzanka, \& MacKie, 2011). Regarding the type of chocolate, 272 white and milk chocolate are mainly made from cocoa butter, an extract from cocoa liquor, containing phenolic 273 compounds in less proportion. In contrast, dark chocolate is mainly made with cocoa liquor what is reflected 274 in the results regarding to the polyphenols content and final bioaccessibility. Figure 2 details the release and solubilisation of phenolic compounds (mg equivalent GA / $\mathrm{g}$ of chocolate), depending on the amount of pancreatin (0-3,000 LU/g fat), at constant conditions of $\mathrm{pH}(6)$ and biliary concentration (1 $\mathrm{mM})$. A positive effect of the presence of pancreatin on the soluble content of polyphenols was observed. According to these results, the oral supplementation with encapsulated pancreatin seems to be crucial for polyphenols release under EPI conditions. The amount of pancreatin, however, was only relevant for the polyphenols release with digested dark chocolate. The absence of dairy compounds in dark chocolate might be favourable, given that a negative effect of caseins on polyphenols release has been reported (Keogh, Mclnerney, \& Clifton, 2007). Their antioxidant activity seems to be also compromised in presence of $\alpha$-casein (Bourassa, Côté, Hutchandani, Samson, \& Tajmir-Riahi, 2013) and milk $\beta$-lactoglobulin by the formation of weak bonds between these proteins and polyphenols (Kanakis et al., 2011), which is the case for milk and white chocolate. Previous studies have demonstrated the reduction of the antioxidant potential by the union of phenolic compounds with milk proteins, reducing their accessibility (Kilmartin \& Hsu, 2003; O'Connell, Fox, Tan-Kintia, \& Fox, 1998). Some previous studies have highlighted a positive correlation between the percentage of cocoa solids identified in the labelling and the concentration of polyphenols (Vinson \& Motisi, 2015). This information could allow consumers identifying those chocolates with greater content of pure cocoa which directly affects the bioavailability of its antioxidants. The above mentioned relation has been highlighted in previous studies (Ortega et al., 2009) in which it is emphasized that a higher content of fat in the digested fluids, favours the formation of emulsified fat drops and with it, the incorporation of cocoa phenols in the lipid phase, protecting them during the duodenal stage. It is also important to emphasize that the consumption of polyphenols in pieces of dark chocolate produces a post-prandial anti-oxidant effect in vivo human despite the presence of fats and sugars in the product (Keen et al., 2005; Ortega et al., 2009).

\subsection{Lipolysis extent affected by chocolate type and intestinal conditions}

During digestion chocolate is mixed with digestive fluids and so the hydration of the matrix occurs, leading this to the solubilisation of some components such as sugars, together with the melting and emulsification of fat. Additionally, the digestive enzymes activity is responsible of macronutrients hydrolysis, and due to the high fat content of chocolates, lipolysis extent after the in vitro digestion process was analysed. Since fat digestion mainly takes place at the duodenum, and because it can be affected by $\mathrm{pH}$, bile concentration and pancreatic enzyme activity in the case of exocrine pancreatic insufficiency, different conditions were analysed. Figure $3 \mathrm{~A}$ 
shows the concentration of FFA/g of fat in the digested samples at different intestinal conditions. These results reveal that the influence of intestinal $\mathrm{pH}$ and bile concentration depends on the type of chocolate; while milk chocolate lipolysis does not depend on $\mathrm{pH}$ or bile concentration, a significant influence of both factors was found for both, dark and white chocolate lipolysis, being more significant in the case of white chocolate. These results are related with the above-mentioned factors affecting MDI and polyphenols release, which are also involved in lipolysis extent during digestion. Food lipids have to be accessible to the digestive enzymes and especially to pancreatic lipase, so it would be expected that those samples with lower MDI would be the ones presenting lower progress of lipolysis. This is the case of dark chocolate samples, which reached the lowest values of FFA/g of fat after digestion independently of the intestinal conditions applied during the in vitro digestion. The more aggregated structure of this type of chocolate makes difficult, not only the matrix disintegration, but also the fat digestion. On the contrary, for milk chocolate, which has a less aggregated structure with more open spaces (filled with fats) than dark chocolate, higher values of lipolysis were obtained. Additionally, the milk proteins that characterize milk chocolate are mainly $80 \%$ of caseins and $20 \%$ of whey proteins. The casein fraction acts as surfactant reducing the viscosity of the chocolate (Afoakwa, Paterson, \& Fowler, 2007) and this decrease in viscosity improves lipid digestibility (Guo, Ye, Bellissimo, Singh, \& Rousseau, 2017); and thus the effect of biliary salts and intestinal pH is not as important as in the case of the other two types of chocolate.

In order to analyse the influence of pancreatic enzyme concentration on lipolysis extent, chocolate samples were in vitro digested in presence of different LU per gram of fat. The most critical intestinal conditions were applied, mimicking the exocrine pancreatic insufficiency ( $\mathrm{pH} 6$ and bile $1 \mathrm{mM}$ ) (Figure 3B). No significant differences were found between the enzyme amount used above and below the recommendation $(2,000 \mathrm{LU} / \mathrm{g}$ fat), but it draws attention that white chocolate reached lower values of lipolysis extent than the milk one, in spite of being the samples with the highest MDI. These results can be explained comparing the formulation of both types of chocolate, and especially those ingredients acting as emulsifiers since they usually play a key role during lipids digestion. The effect of interfacial molecules on lipolysis has been well described in the literature. Borgström, \& Erlanson (1973) and Borgström \& Erlanson (1978) found that high surfactant concentration inhibited lipolysis even in the presence of colipase, and postulated that the bile salts desorbed the surfactants allowing lipase adsorption in the presence of colipase. The low bile concentration used in this work (to mimic abnormal conditions in some chronic diseases such in Cystic Fibrosis) together with the presence of certain emulsifiers would explain the obtained results of FFA. E476 is a synthetic vegetable fat obtained by a combination of polyglycerol and castor oil, which has been reported in the literature to delay lipolysis (Shima, Tanaka, Kimura, Adachi, \& Matsuno, 2004). It is worth noting, that polyglycerol 
polyrincinoleate (E476) is found among the emulsifiers included in white chocolate formula, while it is not included in dark and milk chocolate. In the case of dark chocolate, it is also important to highlight the effect of antioxidants on lipid digestibility. The polyphenols affect the emulsion that occurs during the digestive transit, increasing the particle size and decreasing the specific surface area, causing a decrease in the activity of the lipase (Sugiyama et al., 2007; Uchiyama et al., 2011). Thus, small differences in the formulation of chocolates, especially those with emulsification properties, can have a significant impact on fat digestion, especially under abnormal conditions of bile secretion.

\section{Conclusions}

As the results have shown, we can conclude that it is the type of chocolate the main factor affecting matrix degradation index during digestion. The different ingredients and their proportion that conform the food matrix, like fat, emulsifiers, and crystalline lactose, have a significant impact on the structure and consequently their matrix degradation during digestion.

Under EPI conditions it is important the supplementation of pancreatin for polyphenols release, especially in the case of dark chocolate, which is the one with the highest polyphenols content. A slight increase of polyphenols on the liquid phase for the different chocolates was found by increasing the bile concentration at low $\mathrm{pH}(6)$; while no influence of biliary salts was found at higher intestinal $\mathrm{pH}$ (7). Additionally, a negative effect in the antioxidant activity due to the dairy compounds in milk and white chocolate was observed.

Regarding the influence of different intestinal conditions on lipolysis extent, $\mathrm{pH}$ and bile concentration do not determine the extent of lipolysis in milk chocolate, nevertheless, an influence of both factors was found in both, dark and white chocolate lipolysis. No significant differences were observed between the different amounts of pancreatin used below or above the recommended one $(2,000 \mathrm{LU} / \mathrm{g}$ fat). If we speak about lipolysis, it is important to consider those factors that, due to the nature of the matrix, will affect the results. This is how the presence of emulsifiers in the case of white chocolate, or the presence of antioxidants in dark chocolate determined a decrease in lipolysis.

To sum up, the results obtained in the present study highlight the influence that not only food related factors such food composition, food matrix, processing conditions etc., but also individual physiological parameters (intestinal $\mathrm{pH}$ and bile concentration) might have on nutrients release and bioaccessibility. However, in vivo studies are advisable to be conducted in order to complement these findings and assist food industry in developing tailor-made foodstuffs and to help health professional and dieticians to adapt the existing nutritional guidelines addressed to specific target populations in which intestinal conditions could be altered. 
There are no conflicts of interest to declare.

368

\section{Acknowledgements}

370 371 372 373 374 375 376

Authors of this paper, on behalf of MyCyFAPP consortium, acknowledge the European Union and the Horizon 2020 Research and Innovation Framework Programme for funding the above-mentioned project under grant agreement number 643806. The authors would like to thank the Secretaría de Educación Superior, Ciencia, Tecnología e Innovación (Ecuador) for the PhD scholarship given to Carolina Alicia Paz Yépez.

\section{References}

Afoakwa, E. O., Paterson, A., \& Fowler, M. (2007). Factors influencing rheological and textural qualities in chocolate - a review. Trends in Food Science and Technology, 18(6), 290-298.

Afoakwa, E. O., Paterson, A., Fowler, M., \& Vieira, J. (2009). Microstructure and mechanical properties related to particle size distribution and composition in dark chocolate. International Journal of Food Science and Technology, 44(1), 111-119.

Almoosawi, S., Tsang, C., Ostertag, L. M., Fyfe, L., \& Al-Dujaili, E. A. S. (2012). Differential effect of polyphenol-rich dark chocolate on biomarkers of glucose metabolism and cardiovascular risk factors in healthy, overweight and obese subjects: a randomized clinical trial. Food \& Function, 3(10), 1035.

Asensio-Grau, A., Peinado, I., Heredia, A., \& Andrés, A. (2018). Effect of cooking methods and intestinal conditions on lipolysis, proteolysis and xanthophylls bioaccessibility of eggs. Journal of Functional Foods, 46, 579-586.

Beckett, S. T. (2008). The Science of chocolate. The Royal Society of Chemistry (2nd. Ed). York, UK.

Borgström, B., \& Erlanson, C. (1973). Pancreatic Lipase and Co-Lipase: Interactions and effects of bile salts and other detergents. European Journal of Biochemestry, 37(1), 60-68. https://doi.org/10.1002/eqe.418

Borgström, B., \& Erlanson, C. (1978). Interactions of serum albumin and other proteins with porcine pancreatic lipase. Gastroenterology, 75(3), 382-386.

Bourassa, P., Côté, R., Hutchandani, S., Samson, G., \& Tajmir-Riahi, H. A. (2013). The effect of milk alphacasein on the antioxidant activity of tea polyphenols. Journal of Photochemistry and Photobiology B: Biology, 128, 43-49.

Carrière, F., Renou, C., Lopez, V., De Caro, J., Ferrato, F., Lengsfeld, H., ... Verger, R. (2000). The specific activities of human digestive lipases measured from the in vivo and in vitro lipolysis of test meals. 
Cooper, K. A., Donovan, J. L., Waterhouse, A. I., \& Williamson, G. (2008). Cocoa and health: A decade of research. British Journal of Nutrition, 99(1), 1-11.

401

402

403

404

405

406

407

408

409

410

Faulks, R. M., \& Southon, S. (2005). Challenges to understanding and measuring carotenoid bioavailability. Biochimica et Biophysica Acta - Molecular Basis of Disease, 1740(2), 95-100.

Gelfond, D., Ma, C., Semler, J., \& Borowitz, D. (2013). Intestinal ph and gastrointestinal transit profiles in cystic fibrosis patients measured by wireless motility capsule. Digestive Diseases and Sciences, 58(8), 2275-2281.

Glicerina, V., Balestra, F., Dalla Rosa, M., \& Romani, S. (2016). Microstructural and rheological characteristics of dark, milk and white chocolate: A comparative study. Journal of Food Engineering, $169,165-171$.

González-Bacerio, J., Rodríguez Hernández, J., \& del Monte Martínez, A. (2010). Lipases: enzymes with potential for the development of immobilized biocatalysts by interfacial adsorption. Revista Colombiana de Biotecnología, 12(1), 113-140.

Granado-Lorencio, F., Olmedilla-Alonso, B., Herrero-Barbudo, C., Pérez-Sacristán, B., Blanco-Navarro, I., \& Blázquez-García, S. (2007). Comparative in vitro bioaccessibility of carotenoids from relevant contributors to carotenoid intake. Journal of Agricultural and Food Chemistry, 55(15), 6387-6394.

Guo, Q., Ye, A., Bellissimo, N., Singh, H., \& Rousseau, D. (2017). Modulating fat digestion through food structure design. Progress in Lipid Research, 68(August), 109-118.

Hunter, E. J. (2001). Studies on effects of dietary fatty acids as related to their position on triglycerides. Lipids, 36(7), 655-668.

Jakobek, L. (2015). Interactions of polyphenols with carbohydrates, lipids and proteins. Food Chemistry, 175, $556-567$.

Jalil, A. M. M., \& Ismail, A. (2008). Polyphenols in cocoa and cocoa products: Is there a link between antioxidant properties and health? Molecules, 13(9), 2190-2219.

Kanakis, C. D., Hasni, I., Bourassa, P., Tarantilis, P. A., Polissiou, M. G., \& Tajmir-Riahi, H. A. (2011). Milk $\beta$ lactoglobulin complexes with tea polyphenols. Food Chemistry, 127(3), 1046-1055.

Keen, C. L., Holt, R. R., Oteiza, P. I., Fraga, C. G., \& Schmitz, H. H. (2005). Cocoa antioxidants and cardiovascular health. The American Journal of Clinical Nutrition, 81(1 Suppl), 298-303.

Keogh, J. B., McInerney, J., \& Clifton, P. M. (2007). The effect of milk protein on the bioavailability of cocoa polyphenols. Journal of Food Science, 72(3). https://doi.org/10.1111/j.1750-3841.2007.00314.x

Kilmartin, P. A., \& Hsu, C. F. (2003). Characterisation of polyphenols in green, oolong, and black teas, and in 
coffee, using cyclic voltammetry. Food Chemistry, 82(4), 501-512.

Kong, F., \& Singh, R. P. (2009). Digestion of raw and roasted almonds in simulated gastric environment. Food Biophysics, 4(4), 365-377.

Kurosawa, T., Itoh, F., Nozaki, A., Nakano, Y., Katsuda, S. I., Osakabe, N., ... Itakura, H. (2005). Suppressive effects of cacao liquor polyphenols (CLP) on LDL oxidation and the development of atherosclerosis in Kurosawa and Kusanagi-hypercholesterolemic rabbits. Atherosclerosis, 179(2), 237246.

Lamothe, S., Corbeil, M.-M., Turgeon, S. L., \& Britten, M. (2012). Influence of cheese matrix on lipid digestion in a simulated gastro-intestinal environment. Food \& Function, 3(7), 724.

Li, L., \& Somerset, S. (2014). Digestive system dysfunction in cystic fibrosis: Challenges for nutrition therapy. Digestive and Liver Disease, 46(10), 865-874.

Maldonado-Valderrama, J., Wilde, P., Maclerzanka, A., \& MacKie, A. (2011). The role of bile salts in digestion. Advances in Colloid and Interface Science, 165(1), 36-46.

Matsui, N., Ito, R., Nishimura, E., Yoshikawa, M., Kato, M., Kamei, M., ... Hashizume, S. (2005). Ingested cocoa can prevent high-fat diet-induced obesity by regulating the expression of genes for fatty acid metabolism. Nutrition, 21(5), 594-601.

Minekus, M., Alminger, M., Alvito, P., Ballance, S., Bohn, T., Bourlieu, C., ... Brodkorb, A. (2014). A standardised static in vitro digestion method suitable for food - an international consensus. Food \& Function, 5(5), 1113-1124.

Nimalaratne, C., Savard, P., Gauthier, S. F., Schieber, A., \& Wu, J. (2015). Bioaccessibility and Digestive Stability of Carotenoids in Cooked Eggs Studied Using a Dynamic in Vitro Gastrointestinal Model. Journal of Agricultural and Food Chemistry, 63(11), 2956-2962.

O'Connell, J. E., Fox, P. D., Tan-Kintia, R., \& Fox, P. F. (1998). Effects of tea, coffee and cocoa extracts on the colloidal stability of milk and concentrated milk. International Dairy Journal, 8(8), 689-693.

Ortega, N., Reguant, J., Romero, M. P., Macià, A., \& Motilva, M. J. (2009). Effect of fat content on the digestibility and bioaccessibility of cocoa polyphenol by an in vitro digestion model. Journal of Agricultural and Food Chemistry, 57(13), 5743-5749.

Peinado, I., Larrea, V., Heredia, A., \& Andrés, A. (2018). Lipolysis kinetics of milk-fat catalyzed by an enzymatic supplement under simulated gastrointestinal conditions. Food Bioscience, 23, 1-8.

Pineda-Vadillo, C., Nau, F., Guerin-Dubiard, C., Jardin, J., Lechevalier, V., Sanz-Buenhombre, M., ... Dupont, D. (2017). The food matrix affects the anthocyanin profile of fortified egg and dairy matrices during processing and in vitro digestion. Food Chemistry, 214, 486-496. 
Prazeres, D. M. F., Garcia, F. A. P., \& Cabral, M. S. (1994). Continuous lipolysis in a reversed micellar membrane bioreactor. Bioprocess Engineering, 10, 21-27.

Ramiro, E., Franch, À., Castellote, C., Andrés-Lacueva, C., Izquierdo-Pulido, M., \& Castell, M. (2005). Effect of Theobroma cacao flavonoids on immune activation of a lymphoid cell line. British Journal of Nutrition, 93(6), 859.

Rodríguez-Roque, M. J., de Ancos, B., Sánchez-Moreno, C., Cano, M. P., Elez-Martínez, P., \& MartínBelloso, O. (2015). Impact of food matrix and processing on the in vitro bioaccessibility of vitamin C, phenolic compounds, and hydrophilic antioxidant activity from fruit juice-based beverages. Journal of Functional Foods, 14, 33-43.

Rovner, A. J., Schall, J. I., Mondick, J. T., Zhuang, H., \& Mascarenhas, M. R. (2013). Delayed small bowel transit in children with cystic fibrosis and pancreatic insufficiency. Journal of Pediatric Gastroenterology and Nutrition, 57(1), 81-84.

Ryan, L., O’Connell, O., O’Sullivan, L., Aherne, S. A., \& O’Brien, N. M. (2008). Micellarisation of carotenoids from raw and cooked vegetables. Plant Foods for Human Nutrition, 63(3), 127-133.

Shani-Levi, C., Alvito, P., Andrés, A., Assunção, R., Barberá, R., Blanquet-Diot, S., ... Lesmes, U. (2017). Extending in vitro digestion models to specific human populations: Perspectives, practical tools and biorelevant information. Trends in Food Science \& Technology, 60, 52-63.

Shima, M., Tanaka, M., Kimura, Y., Adachi, S., \& Matsuno, R. (2004). Hydrolysis of the oil phase of a W/O/W emulsion by pancreatic lipase. Journal of Controlled Release, 94(1), 53-61.

Shishikura, Y., Khokhar, S., \& Murray, B. S. (2006). Effects of tea polyphenols on emulsification of olive oil in a small intestine model system. Journal of Agricultural and Food Chemistry, 54(5), 1906-1913.

Singleton, V. L., Orthofer, R., \& Lamuela-Raventós, R. M. (1998). Analysis of total phenols and other oxidation substrates and antioxidants by means of folin-ciocalteu reagent. Methods in Enzymology, 299(1974), 152-178.

Sugiyama, H., Akazome, Y., Shoji, T., Yamaguchi, A., Yasue, M., Kanda, T., \& Ohtake, Y. (2007). Oligomeric procyanidins in apple polyphenol are main active components for inhibition of pancreatic lipase and triglyceride absorption. Journal of Agricultural and Food Chemistry, 55(11), 4604-4609.

Tarko, T., Duda-Chodak, A., \& Zajac, N. (2013). Digestion and absorption of phenolic compounds assessed by in vitro simulation methods. A review. Roczniki Państwowego Zakładu Higieny, 64(2), 79-84.

Turck, D., Braegger, C. P., Colombo, C., Declercq, D., Morton, A., Pancheva, R., ... Wilschanski, M. (2016). ESPEN-ESPGHAN-ECFS guidelines on nutrition care for infants, children, and adults with cystic fibrosis. Clinical Nutrition, 35(3), 557-577. 
494 Uchiyama, S., Taniguchi, Y., Saka, A., Yoshida, A., \& Yajima, H. (2011). Prevention of diet-induced obesity 495 by dietary black tea polyphenols extract in vitro and in vivo. Nutrition, 27(3), 287-292.

496 Vinson, J. A., \& Motisi, M. J. (2015). Polyphenol antioxidants in commercial chocolate bars: Is the label 497 accurate? Journal of Functional Foods, 12, 526-529.

498 Vinson, J. A., Proch, J., Bose, P., Muchler, S., Taffera, P., Shuta, D., ... Agbor, G. A. (2006). Chocolate is a 499 powerful ex vivo and in vivo antioxidant, an antiatherosclerotic agent in an animal model, and a 500 significant contributor to antioxidants in the European and American diets. Journal of Agricultural and $501 \quad$ Food Chemistry, 54(21), 8071-8076.

502 Whitcomb, D. C., Lehman, G. A., Vasileva, G., Malecka-Panas, E., Gubergrits, N., Shen, Y., ... Caras, S. 503 (2010). Pancrelipase delayed-release capsules (CREON) for exocrine pancreatic insufficiency due to 504 chronic pancreatitis or pancreatic surgery: A double-blind randomized trial. American Journal of 505 Gastroenterology, 105(10), 2276-2286.

506 Wollgast, J., \& Anklam, E. (2000). Review on polyphenols in Theobroma cacao: Changes in composition 507 during the manufacture of chocolate and methodology for identification and quantification. Food $508 \quad$ Research International, 33(6), 423-447. 
511

512

513

514

515

516

517

518

519

520

521

522

523

524

525

526

527

528

529

530

531

532

533

534

535

536

537

538

539

540

Figure Captions:

Figure 1. Polyphenols content (mg Gallic Acid eq/ gram of chocolate) along the in vitro digestion of different type of chocolates (dark, milk and white) at intestinal conditions of $\mathrm{pH} 6$ or 7 , bile concentration of 1 or $10 \mathrm{mM}$ and at a fixed pancreatic enzyme amount of 2,000 LU/g fat. Polyphenols content has been measured in chocolate before digestion (discontinuous line), and in both, liquid and solid phases, in digested chocolate.

Letters a-e refer to the homogenous groups obtained by the Fisher LSD post-hoc test after the ANOVA applied to data to each chocolate type, they provide information about the effect of the different digestion stages on the release of polyphenols available for absorption ( $p$-value $<0.05)$.

Figure 2. Polyphenols content (mg Gallic Acid eq/ gram of chocolate) after in vitro digestion of different type of chocolates (dark, milk and white), digested under fixed intestinal conditions ( $\mathrm{pH} 6$ and bile concentration 1 $\mathrm{mM}$ ) and different pancreatin concentrations (0-3,000 LU/g fat). (A) Values from liquid phase (B) values from solid phase.

Letters a-c refer to the homogeneous groups obtained by the Fisher LSD post-hoc test after the ANOVA applied to data within each single type of chocolate and they provide information about the release of polyphenols under different pancreatin concentrations. Letters A-C refer to the homogenous groups obtained by the Fisher LSD post-hoc test after the ANOVA applied to data between all type of chocolates for each single pancreatin concentration; thus they provide information related to the effect of the type of chocolate on the release of polyphenols ( $p$-value $<0.05)$.

Figure 3. A) Lipolysis (mg FFA/g fat) after the in vitro digestion with a fixed pancreatin concentration (2000 $\mathrm{LU} / \mathrm{g}$ fat) and different combinations of intestinal $\mathrm{pH}$ and bile concentrations ( $\mathrm{pH} 6$ or 7 , bile salts concentration 1 or $10 \mathrm{mM}$ ); B) Lipolysis (mg FFA/g fat) after the in vitro digestion under fixed intestinal conditions (pH 6 and bile concentration $1 \mathrm{mM})$ and different pancreatin concentrations (0-3,000 LU/g fat).

Letters (a-b "lower case") refer to the homogenous groups obtained by the Fisher LSD post-hoc test after the ANOVA applied comparing between different lipase concentrations or intestinal conditions within the same type of chocolate, and letters (A-C "capital letters") refer to the homogenous groups obtained by the Fisher 
541 LSD post-hoc test after the ANOVA applied comparing between different type of chocolates for each 542 experimental condition ( $p$-value $<0.05$ ).

543 